\title{
New Block Triangular Preconditioners for Saddle Point Linear Systems with Highly Singular $(1,1)$ Blocks
}

\section{TingZhu Huang, GuangHui Cheng, and Liang Li}

School of Applied Mathematics/Institue of Computational Science, University of Electronic Science and Technology of China, Chengdu, Sichuan 610054, China

Correspondence should be addressed to GuangHui Cheng, ghcheng@uestc.edu.cn

Received 10 December 2008; Revised 29 March 2009; Accepted 6 August 2009

Recommended by Victoria Vampa

We establish two types of block triangular preconditioners applied to the linear saddle point problems with the singular $(1,1)$ block. These preconditioners are based on the results presented in the paper of Rees and Greif (2007). We study the spectral characteristics of the preconditioners and show that all eigenvalues of the preconditioned matrices are strongly clustered. The choice of the parameter is involved. Furthermore, we give the optimal parameter in practical. Finally, numerical experiments are also reported for illustrating the efficiency of the presented preconditioners.

Copyright (c) 2009 TingZhu Huang et al. This is an open access article distributed under the Creative Commons Attribution License, which permits unrestricted use, distribution, and reproduction in any medium, provided the original work is properly cited.

\section{Introduction}

Consider the following saddle point linear system:

$$
\mathcal{A} \xi \equiv\left(\begin{array}{cc}
G & B^{T} \\
B & 0
\end{array}\right)\left(\begin{array}{l}
x \\
y
\end{array}\right)=\left(\begin{array}{l}
b \\
q
\end{array}\right) \equiv f,
$$

where $G \in \mathbb{R}^{n \times n}$ is a symmetric and positive semidefinite matrix with nullity (=dim $(\operatorname{kernel}(G))) p$, the matrix $B \in \mathbb{R}^{m \times n}$ has full row rank, vectors $x, b \in \mathbb{R}^{n}$, and vectors $y, q \in \mathbb{R}^{m}$, and vectors $x, y$ are unknown. The assumption that $\mathcal{A}$ is nonsingular implies that null $(G) \cap$ $\operatorname{null}(B)=\{0\}$, which we use in the following analysis. Under these assumptions, the system (1.1) has a unique solution. This system is very important and appears in many different applications of scientific computing, such as constrained optimization $[1,2]$, the finite element method for solving the Navier-Stokes equation [3-6], fluid dynamics, constrained least problems and generalized least squares problems [7-10], and the discretized timeharmonic Maxwell equations in mixed form [11]. 
Recently, T. Rees and C. Greif explored a preconditioning technique applied to the problem of solving linear systems arising from primal-dual interior point algorithms and quadratic programming in [12]. The preconditioner has the attractive property of improved eigenvalue clustering with increasing ill-conditioned $(1,1)$ block of the symmetric saddle point systems. To solve the saddle point system (1.1), Krylov subspace methods are usually used in modern solution techniques which rely on the ease of sparse matrix-vector products and converges at a rate dependent on the number of distinct eigenvalues of the preconditioned matrix $[13,14]$.

The rest of this paper, two types of block triangular preconditioners are established for the saddle point systems with an ill-conditioned $(1,1)$ block. Our methodology extends the recent work done by Greif and Schötzau [11, 15], and Rees and Greif [12].

This paper is organized as follows. In Section 2, we will establish new precondtioners and study the spectral analysis of the new preconditioners for the saddle point system. Some numerical examples are given in Section 3. Finally, conclusions are made in Section 4.

\section{Preconditioners and Spectrum Analysis}

For linear systems, the convergence of an applicable iterative method is determined by the distribution of the eigenvalues of the coefficient matrix. In particular, it is desirable that the number of distinct eigenvalues, or at least the number of clusters, is small, because in this case convergence will be rapid. To be more precise, if there are only a few distinct eigenvalues, then optimal methods like BiCGStab or GMRES will terminate (in exact arithmetic) after a small and precisely defined number of steps.

Rees and Greif [12] established the following preconditioner for the symmetric saddle point system (1.1):

$$
\mathcal{M}=\left(\begin{array}{cc}
G+B^{T} W^{-1} B & t B^{T} \\
0 & W
\end{array}\right)
$$

where $t$ is a scalar and $W$ is an $m \times m$ symmetric positive weight matrix. Similar to $\mathcal{M}$, we introduce the following precondtioners for solving symmetric saddle point systems:

$$
\mathcal{M}_{t}=\left(\begin{array}{cc}
G+B^{T} W^{-1} B & (1-t) B^{T} \\
0 & t W
\end{array}\right),
$$

where $t \neq 0$ is a parameter, and

$$
\widehat{M}_{t}=\left(\begin{array}{cc}
G+t B^{T} W^{-1} B & t B^{T} \\
0 & \frac{1-t}{t} W
\end{array}\right)
$$

where $1 \neq t>0$. 
Theorem 2.1. The matrix $\mathfrak{M}_{t}^{-1} \mathbb{A}$ has two distinct eigenvalues which are given by

$$
\lambda_{1}=1, \quad \lambda_{2}=-\frac{1}{t}
$$

with algebraic multiplicity $n$ and $p$, respectively. The remaining $m-p$ eigenvalues satisfy the relation

$$
\lambda=\frac{-\mu}{t(\mu+1)}
$$

where $\mu$ are some $m$ - $p$ generalized eigenvalues of the following generalized eigenvalue problem:

$$
B^{T} W^{-1} B x=\mu G x .
$$

Let $\left\{z_{i}\right\}_{i=1}^{n-m}$ be a basis of the null space of $B$, let $\left\{u_{i}\right\}_{i=1}^{p}$ be a basis of the null space of $G$, and $\left\{v_{i}\right\}_{i=1}^{m-p}$ be a set of linearly independent vectors that complete null $(G) \cup$ null $(B)$ to a basis of $\mathbb{R}^{n}$. Then the vectors $\left[z_{i}^{T}, 0^{T}\right]^{T}(i=1, \ldots, n-m)$, the vectors $\left[u_{i}^{T},(1 / t)\left(W^{-1} B u_{i}\right)^{T}\right]^{T}(i=1, \ldots, p)$, and the vectors $\left[v_{i}^{T},(1 / t)\left(W^{-1} B v_{i}\right)^{T}\right]^{T}(i=1, \ldots, m-p)$, are linearly independent eigenvectors associated with $\lambda=1$, and the vectors $\left[u_{i}^{T},-\left(W^{-1} B u_{i}\right)^{T}\right]^{T}(i=1, \ldots, p)$ are linearly independent eigenvectors associated with $\lambda=-1 / t$.

Proof. Suppose that $\lambda$ is an eigenvalue of $\mathcal{M}_{t}^{-1} \mathcal{A}$, whose eigenvector is $\left(\begin{array}{l}x \\ y\end{array}\right)$. So, we have

$$
\mathcal{M}_{t}^{-1} A\left(\begin{array}{l}
x \\
y
\end{array}\right)=\lambda\left(\begin{array}{l}
x \\
y
\end{array}\right)
$$

Furthermore, it satisfies the generalized eigenvalue problem

$$
\left(\begin{array}{cc}
G & B^{T} \\
B & 0
\end{array}\right)\left(\begin{array}{l}
x \\
y
\end{array}\right)=\lambda\left(\begin{array}{cc}
G+B^{T} W^{-1} B & (1-t) B^{T} \\
0 & t W
\end{array}\right)\left(\begin{array}{l}
x \\
y
\end{array}\right) .
$$

The second block row gives $y=(1 / \lambda t) W^{-1} B x$, substituting which into the first block row equation gives

$$
(\lambda-1)\left[\lambda G x+\left(\lambda+\frac{1}{t}\right) B^{T} W^{-1} B x\right]=0 .
$$

By inspection it is straightforward to see that any vector $x \in \mathbb{R}^{n}$ satisfies (2.9) with $\lambda=1$; thus the latter is an eigenvalue of $\mathcal{M}_{t}^{-1} \mathcal{A}$ and $\left[x^{T},(1 / t)\left(W^{-1} B x\right)^{T}\right]^{T}$ is an eigenvector of $\mathcal{M}_{t}^{-1} \mathcal{A}$. We obtain that the eigenvalue $\lambda=1$ has algebraic multiplicity $n$. From the nullity of $G$ it follows that there are $p$ linearly independent null vectors of $G$. For each such null vector $x \in \operatorname{null}(G)$ we can obtain

$$
\lambda=-\frac{1}{t}
$$

each with algebraic multiplicity $p$ and $\left[x^{T},-\left(W^{-1} B x\right)^{T}\right]^{T}$ is an eigenvalue of $\mathcal{M}_{t}^{-1} \mathcal{A}$. 
Let the vectors $\left\{z_{i}\right\}_{i=1}^{n-m}$ be a basis of the null space of $B$, and let $\left\{u_{i}\right\}_{i=1}^{p}$ be a basis of the null space of $G$. Because $\operatorname{null}(G) \cap \operatorname{null}(B)=\{0\}$, the vectors $\left\{z_{i}\right\}_{i=1}^{n-m}$ and $\left\{u_{i}\right\}_{i=1}^{p}$ are linearly independent and together span the subspace null $(G) \cup \operatorname{null}(B)$. Let the vectors $\left\{v_{i}\right\}_{i=1}^{m-p}$ complete $\operatorname{null}(G) \cup \operatorname{null}(B)$ to a basis of $\mathbb{R}^{n}$. It follows that the vectors $\left[z_{i}^{T}, 0^{T}\right]^{T}(i=1, \ldots, n-m)$, the vectors $\left[u_{i}^{T},(1 / t)\left(W^{-1} B u_{i}\right)^{T}\right]^{T}(i=1, \ldots, p)$, and the vectors $\left[v_{i}^{T},(1 / t)\left(W^{-1} B v_{i}\right)^{T}\right]^{T}(i=1, \ldots, m-p)$, are linearly independent eigenvectors associated with $\lambda=1$, and the vectors $\left[u_{i}^{T},-\left(W^{-1} B u_{i}\right)^{T}\right]^{T}(i=1, \ldots, p)$ are linearly independent eigenvectors associated with $\lambda=-1 / t$.

Next, we consider the remaining $m-p$ eigenvalues. Suppose $\lambda \neq 1$ and $\lambda \neq-1 /$ t. From (2.9) we obtain

$$
B^{T} W^{-1} B x=\mu G x
$$

where $\mu=-t \lambda /(t \lambda+1)$, which implies that $\lambda=-\mu /(t(\mu+1))$.

When the parameter $t=-1$, we easily obtain the following corollary from Theorem 2.1.

Corollary 2.2. Let $t=-1$. Then the matrix $\mathcal{M}_{t}^{-1} \mathbb{A}$ has one eigenvalue which is given by $\lambda=1$ with algebraic multiplicity $n+p$. The remaining $m-p$ eigenvalues satisfy the relation

$$
\lambda=\frac{\mu}{\mu+1}
$$

where $\mu$ are some $m$ - $p$ generalized eigenvalues of the following generalized eigenvalue problem:

$$
B^{T} W^{-1} B x=\mu G x
$$

Theorem 2.3. The matrix $\mathfrak{M}_{t}^{-1} \mathcal{A}$ has two distinct eigenvalues which are given by

$$
\lambda_{1}=1, \quad \lambda_{2}=-\frac{1}{t}
$$

with algebraic multiplicity $n$ and $p$, respectively. The remaining $m$ - $p$ eigenvalues lie in the interval

$$
\left(0,-\frac{1}{t}\right) \quad(t<0) \quad \text { or } \quad\left(-\frac{1}{t}, 0\right) \quad(t>0)
$$

Proof. According to Theorem 2.1, we know that the matrix $\mathcal{M}_{t}^{-1} \mathscr{A}$ has two distinct eigenvalues which are given by

$$
\lambda_{1}=1, \quad \lambda_{2}=-\frac{1}{t}
$$

with algebraic multiplicity $n$ and $p$, respectively. 
From (2.9), we can obtain that the remaining $m-p$ eigenvalues satisfy

$$
\lambda=-\frac{u}{t}
$$

where $u=\left\langle B^{T} W^{-1} B x, x\right\rangle /\langle G x, x\rangle+\left\langle B^{T} W^{-1} B x, x\right\rangle \in \mathbb{R}^{+}$, in which $\langle\cdot, \cdot \cdot\rangle$ is the standard Euclidean inner product, $x \notin \operatorname{null}(G)$ and $x \notin \operatorname{null}(B)$. Evidently, we have $0<u<1$. The expression (2.17) gives an explicit formula in terms of the generalized eigenvalues of (2.17) and can be used to identify the intervals in which the eigenvalues lie. Furthermore, we can obtain that the remaining $m-p$ eigenvalues lie in the interval $(0,-1 / t)(t<0)$ or $(-1 / t, 0)(t>0)$.

When the parameter $t=-1$, we easily obtain the following corollary from Theorem 2.3.

Corollary 2.4. Let $t=-1$. Then the matrix $\mathcal{M}_{t}^{-1} \mathbb{A}$ has one eigenvalue which is given by $\lambda=1$ with algebraic multiplicity $n+p$. The remaining $m-p$ eigenvalues lie in the interval $(0,1)$.

Theorem 2.5. The matrix $\widehat{M}_{t}^{-1} \AA$ has two distinct eigenvalues which are given by

$$
\lambda_{1}=1, \quad \lambda_{2}=\frac{1}{t-1}
$$

with algebraic multiplicity $n$ and $p$, respectively. The remaining $m-p$ eigenvalues satisfy the relation

$$
\lambda=\frac{\mu t}{(t-1)(\mu t+1)}
$$

where $\mu$ are some $m$ - p generalized eigenvalues of the following generalized eigenvalue problem:

$$
B^{T} W^{-1} B x=\mu G x
$$

Let $\left\{z_{i}\right\}_{i=1}^{n-m}$ be a basis of the null space of $B$, and let $\left\{u_{i}\right\}_{i=1}^{p}$ be a basis of the null space of $G$, and let $\left\{v_{i}\right\}_{i=1}^{m-p}$ be a set of linearly independent vectors that complete null $(G) \cup \operatorname{null}(B)$ to a basis of $\mathbb{R}^{n}$. Then the vectors $\left[z_{i}^{T}, 0^{T}\right]^{T}(i=1, \ldots, n-m)$, the vectors $\left[u_{i}^{T},(t /(1-t))\left(W^{-1} B u_{i}\right)^{T}\right]^{T}(i=1, \ldots, p)$, and the vectors $\left[v_{i}^{T},(t /(1-t))\left(W^{-1} B v_{i}\right)^{T}\right]^{T}(i=1, \ldots, m-p)$, are linearly independent eigenvectors associated with $\lambda=1$, and the vectors $\left[u_{i}^{T},-t\left(W^{-1} B u_{i}\right)^{T}\right]^{T}(i=1, \ldots, p)$ are linearly independent eigenvectors associated with $\lambda=1 /(t-1)$.

Proof. The proof is similar to the proof of Theorem 2.1.

When the parameter $t=2$, we easily obtain the following corollary from Theorem 2.5. 
Corollary 2.6. Let $t=2$. Then the matrix $\widehat{\mathcal{M}}_{t}^{-1} \mathbb{A}$ has one eigenvalue which is given by

$$
\lambda=1
$$

with algebraic multiplicity $n+p$. The remaining $m-p$ eigenvalues satisfy the relation

$$
\lambda=\frac{2 \mu}{2 \mu+1}
$$

where $\mu$ are some $m$ - p generalized eigenvalues of the following generalized eigenvalue problem:

$$
B^{T} W^{-1} B x=\mu G x
$$

Theorem 2.7. The matrix $\widehat{M}_{t}^{-1} \AA$ has two distinct eigenvalues which are given by

$$
\lambda_{1}=1, \quad \lambda_{2}=\frac{1}{t-1}
$$

with algebraic multiplicity $n$ and $p$, respectively. The remaining $m-p$ eigenvalues lie in the interval

$$
\left(0, \frac{1}{t-1}\right) \quad(t>1) \quad \text { or } \quad\left(\frac{1}{t-1}, 0\right) \quad(t<1)
$$

Proof. The proof is similar to the proof of Theorem 2.3.

When the parameter $t=2$, we easily obtain the following corollary from Theorem 2.7.

Corollary 2.8. Let $t=2$. Then the matrix $\widehat{M}_{t}^{-1} A$ has only one eigenvalue which is given by $\lambda=1$ with algebraic multiplicity $n+p$. The remaining $m-p$ eigenvalues lie in the interval $(0,1)$.

Remark 2.9. The above theorems and corollaries illustrate the strong spectral clustering when the $(1,1)$ block of $A$ is singular. A well-known difficulty is the increasing ill-conditioned $(1$, 1 ) block as the solution is approached. Our claim is that the preconditioners perform robust even as the problem becomes more ill-conditioned; in fact the outer iteration count decreases. On the other hand, solving the augmented $(1,1)$ block may be more computationally difficult and requires effective approaches such as inexact solvers. In Section 3, we indeed consider inexact solvers in numerical experiments.

Remark 2.10. It is clearly seen from Theorems 2.1 and 2.5 and Corollaries 2.2 and 2.6 that our preconditioners are suitable for symmetric saddle point systems, from Theorems 2.3 and 2.7 and Corollaries 2.4 and 2.8 that our preconditioners are most effective than the preconditioner of [12]. 


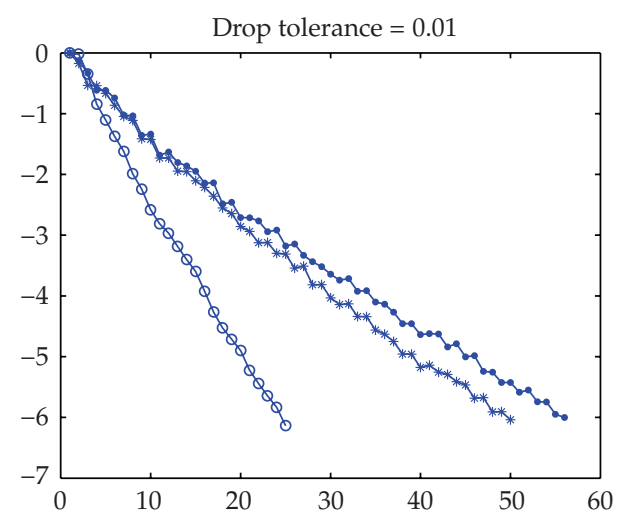

(a)

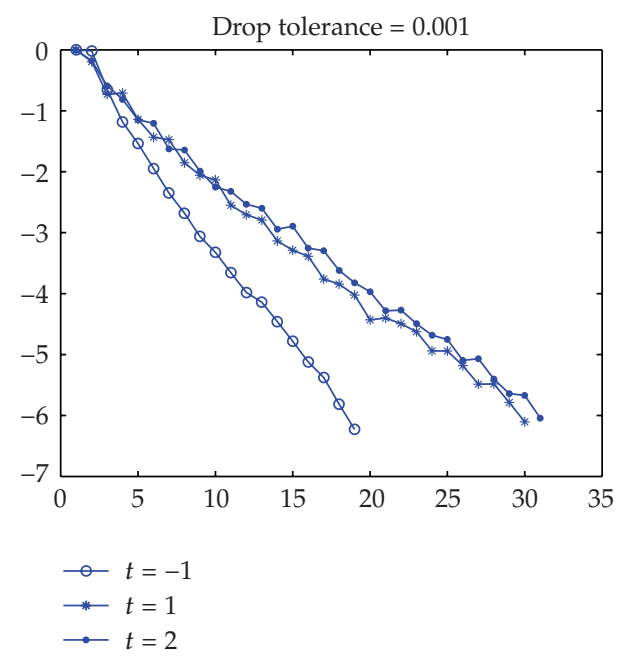

(c)

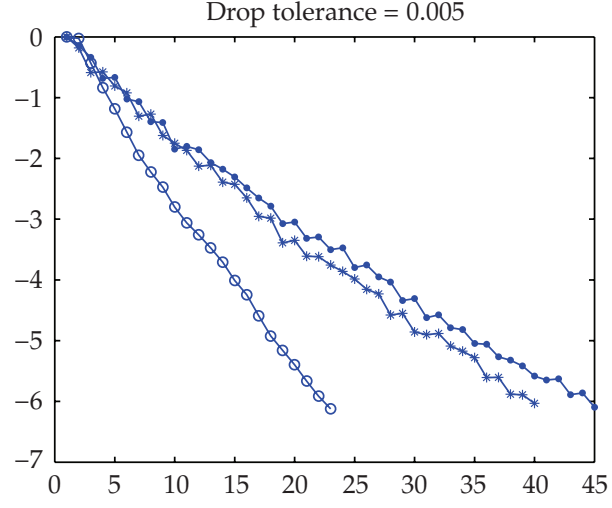

(b)

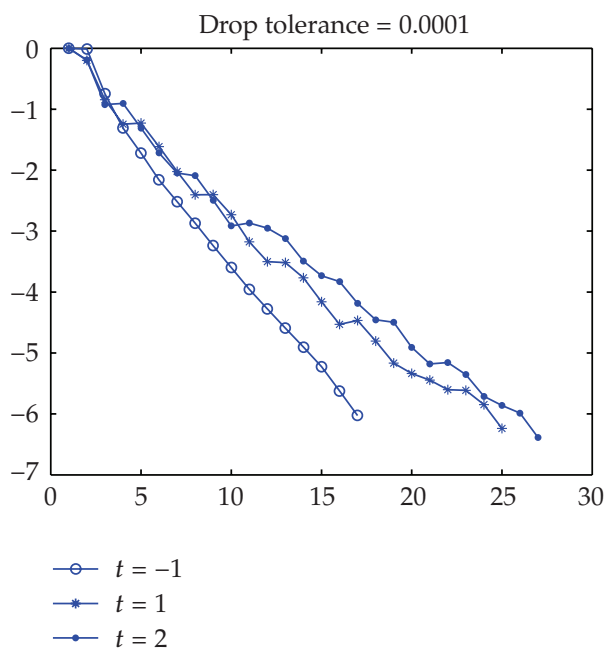

(d)

Figure 1: Convergence curve and total numbers of inner GMRES(10) iterations for different $t$ when $h=$ $1 / 16$.

Remark 2.11. Similarly, the nonsymmetric saddle point linear systems can also obtain the above results.

\section{Numerical Experiments}

All the numerical experiments were performed with MATLAB 7.0. The machine we have used is a PC-Intel(R), Core(TM)2 CPU T7200 2.0 GHz, $1024 \mathrm{M}$ of RAM. The stopping criterion is $\left\|r^{(k)}\right\|_{2} /\left\|r^{(0)}\right\|_{2} \leqslant 10^{-6}$, where $r^{(k)}$ is the residual vector after $k$ th iteration. The right-hand side vectors $b$ and $q$ are taken such that the exact solutions $x$ and $y$ are both vectors with all components being 1 . The initial guess is chosen to be zero vector. We will use preconditioned GMRES(10) to solve the saddle point linear systems. 


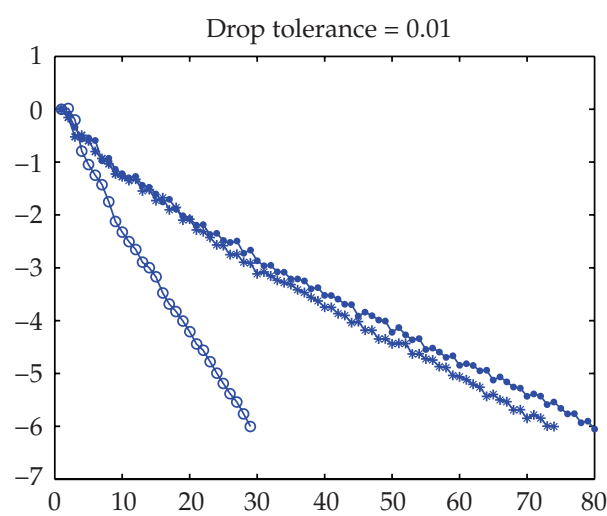

(a)

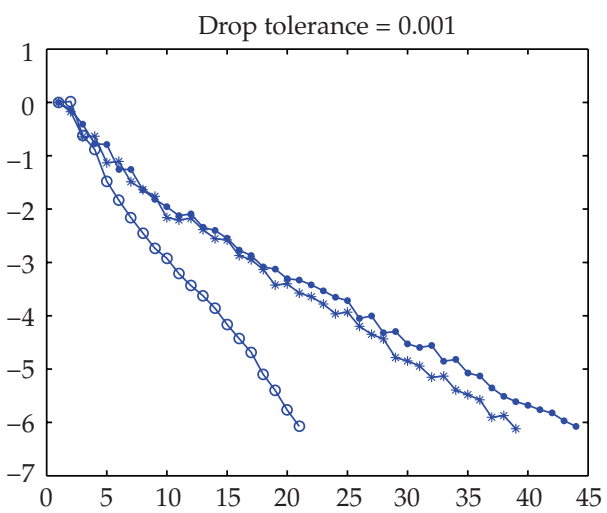

$\rightarrow t=-1$
$\because-t=1$
$\rightarrow t=2$

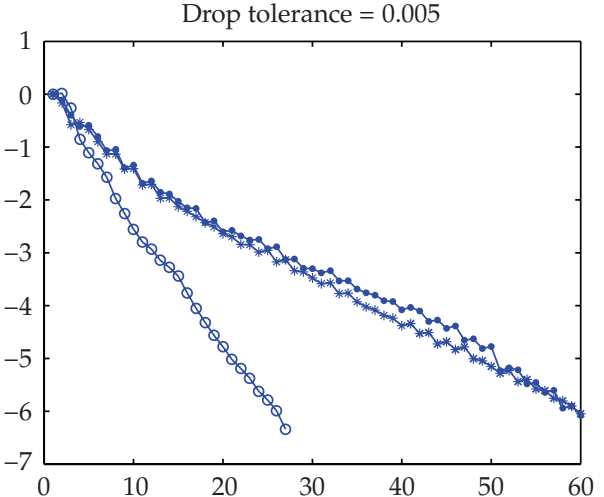

(b)

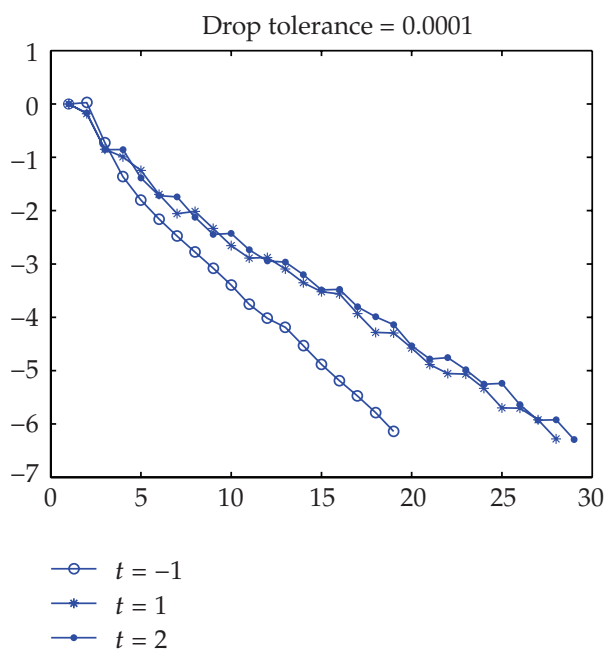

(d)

Figure 2: Convergence curve and total numbers of inner GMRES(10) iterations for different $t$ when $h=$ $1 / 24$.

Our numerical experiments are similar to those in [16]. We consider the matrices taken from [17] with notations slightly changed.

We construct the saddle point-type matrix $\mathcal{A}$ from reforming a matrix $\hat{A}$ of the following form:

$$
\widehat{A}=\left(\begin{array}{ccc}
F_{1} & 0 & B_{u}^{T} \\
0 & F_{2} & B_{v}^{T} \\
B_{u} & B_{v} & 0
\end{array}\right),
$$

where $G \equiv\left(\begin{array}{cc}F_{1} & 0 \\ 0 & F_{2}\end{array}\right)$ is positive real. The matrix $\widehat{A}$ arises from the discretization by the maker and cell finite difference scheme [17] of a leaky two-dimensional lid-driven cavity problem in a square domain $(0 \leq x \leq 1 ; 0 \leq y \leq 1)$. Then the matrix $\left[B_{u}, B_{v}\right]$ is replaced by a random 


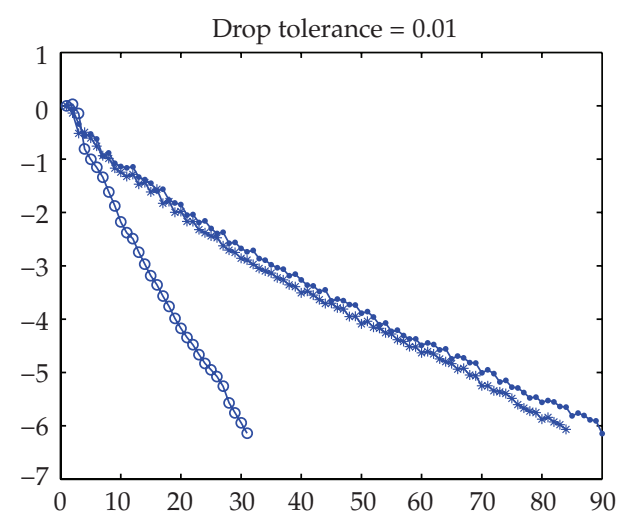

(a)

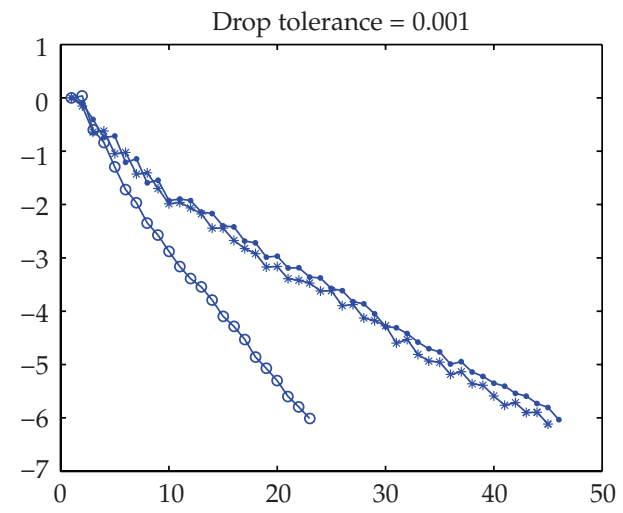

$\longrightarrow t=-1$
$\longrightarrow \quad t=1$
$\longrightarrow t=2$

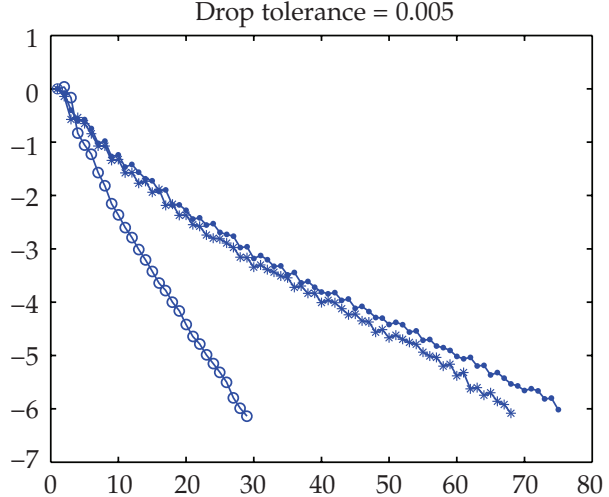

(b)

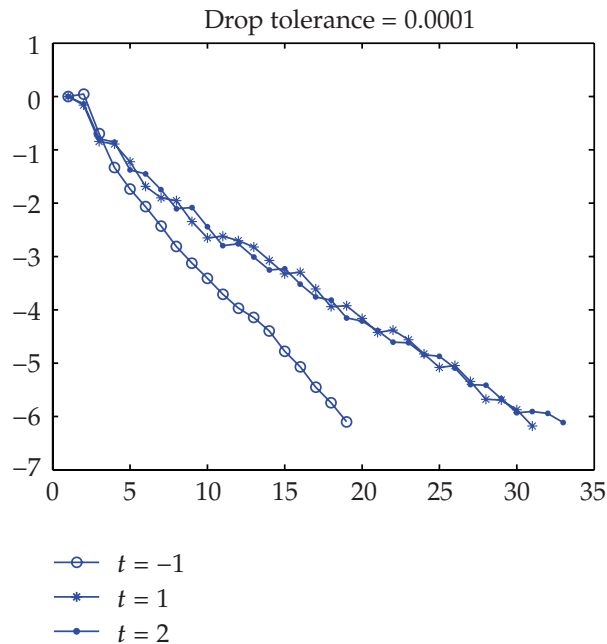

(d)

Figure 3: Convergence curve and total numbers of inner GMRES(10) iterations for different $t$ when $h=$ $1 / 32$.

matrix $\widehat{B}$ with the same sparsity as $\left[B_{u}, B_{v}\right]$, replaced by $B_{1}=\widehat{B}(1: m, 1: m)-(3 / 2) I_{m}$, such that $B_{1}$ is nonsingular. Denote $B_{2}=\widehat{B}(1: m, m+1: n)$, then we have $B=\left[B_{1}, B_{2}\right]$ with $B_{1} \in \mathcal{R}^{m, m}$ and $B_{2} \in \mathcal{R}^{m, n-m}$. Obviously, the resulted saddle point-type matrix

$$
\mathcal{A} \equiv\left(\begin{array}{cc}
G & B^{T} \\
B & 0
\end{array}\right)
$$

satisfies $\operatorname{rank}\left(B^{T}\right)=\operatorname{rank}(B)=m$.

From the matrix $\mathcal{A}$ in (3.2) we construct the following saddle point-type matrix:

$$
\mathcal{A}_{1} \equiv\left(\begin{array}{cc}
G_{1} & B^{T} \\
B & 0
\end{array}\right)
$$


Table 1: Values of $n$ and $m$, and order of $\mathscr{A}_{1}$.

\begin{tabular}{lccc}
\hline$h$ & $n$ & $m$ & Order of $\mathscr{A}_{1}$ \\
\hline $1 / 16$ & 480 & 256 & 736 \\
$1 / 24$ & 1104 & 576 & 1680 \\
$1 / 32$ & 1984 & 1024 & 3008 \\
\hline
\end{tabular}

Table 2: Number and time of iterations of GMRES(10) with preconditioners $\boldsymbol{M}_{t}$ and $\boldsymbol{M}$ for different drop tolerances $\tau$ and $t$ when $h=1 / 16$. Results of preconditioner $\mathcal{M}$ lie inside [ ].

\begin{tabular}{lcccccc}
\hline$\tau$ & $t=-1$ & Time $(-1)$ & $t=1$ & Time $(1)$ & $t=2$ & Time (2) \\
\hline 0.01 & $3(24)$ & 0.1875 & $5(49)$ & 0.3125 & $6(55)$ & 0.3438 \\
& {$[6(58)]$} & {$[0.4219]$} & {$[7(65)]$} & {$[0.4844]$} & {$[10(93)]$} & {$[0.6563]$} \\
0.005 & $3(22)$ & 0.1875 & $4(39)$ & 0.2656 & $5(44)$ & 0.2969 \\
& {$[5(47)]$} & {$[0.3594]$} & {$[6(54)]$} & {$[0.4063]$} & {$[9(85)]$} & {$[0.6406]$} \\
0.001 & $2(18)$ & 0.1719 & $3(29)$ & 0.2188 & $3(30)$ & 0.2188 \\
& {$[4(38)]$} & {$[0.3594]$} & {$[6(52)]$} & {$[0.4688]$} & {$[9(85)]$} & {$[0.7031]$} \\
0.0001 & $2(16)$ & 0.1719 & $3(24)$ & 0.2188 & $3(26)$ & 0.2344 \\
& {$[4(36)]$} & {$[0.3750]$} & {$[5(60)]$} & {$[0.5156]$} & {$[8(73)]$} & {$[0.7188]$} \\
\hline
\end{tabular}

where $G_{1}$ is constructed from $G$ by making its first $m / 4$ rows and columns with zero entries. Note that $G_{1}$ is semipositive definite and its nullity is $m / 4$.

In our numerical experiments the matrix $W$ in the augmentation block preconditioners is taken as $W=I_{m}$. During implementation of our augmentation block preconditioners, we need the operation $\left(G_{1}+B^{T} B\right)^{-1} u$ for a given vector $u$ or, equivalently, need to solve the following equation: $\left(G_{1}+B^{T} B\right) v=u$ for which we use an incomplete LU factorization of $\left(G_{1}+B^{T} B\right)=L U+R$ with drop tolerance $\tau$. Here $m(n)$ means number of outer (inner) iterations. Time $(t)$ represents the corresponding computing time (in seconds) when taking the parameter as $t$.

In the following, we summarize the observations from Tables 1, 2, 3, 4, 5, 6, and 7 and Figures 1, 2, and 3 .

(i) From Tables 2-4, we can find that our preconditioners are more efficient than those of [12] both in number of iterations and time of iterations, especially in the case of the optimal parameter.

(ii) Number and time of iterations with the preconditioner $\mathcal{M}_{-1}$ smaller than those with the preconditioners $\boldsymbol{M}_{1}$ and $\boldsymbol{M}_{2}$. In fact, $\boldsymbol{M}_{1}$ is a diagonal preconditioner.

(iii) Number and time of iterations with the preconditioner $\widehat{\mathbb{M}}_{t}$ are the smallest when $t=2$.

(iv) Number of iterations decreases but the computational cost of incomplete LU factorization increases with decreased $\tau$. Therefore, we should not use the preconditioners with small $\tau$ in practical.

(v) The eigenvalues of $\mathcal{M}_{t}^{-1} \mathcal{A}_{1}$ are strongly clustered. Furthermore, the eigenvalues of $\mathcal{M}_{-1}^{-1} \mathcal{A}_{1}$ are positive. 
Table 3: Number and time of iterations of GMRES(10) with preconditioners $\boldsymbol{M}_{t}$ and $\boldsymbol{M}$ for different drop tolerances $\tau$ and $t$ when $h=1 / 24$. Results of preconditioner $\mathcal{M}$ lie inside [ ].

\begin{tabular}{lcccccc}
\hline$\tau$ & $t=-1$ & Time $(-1)$ & $t=1$ & Time $(1)$ & $t=2$ & Time (2) \\
\hline 0.01 & $3(28)$ & 1.0469 & $8(73)$ & 2.6563 & $8(79)$ & 2.8594 \\
& {$[9(88)]$} & {$[3.3125]$} & {$[9(85)]$} & {$[3.2188]$} & {$[13(125)]$} & {$[4.5469]$} \\
0.005 & $3(26)$ & 1.0000 & $6(59)$ & 2.2969 & $6(59)$ & 2.1719 \\
& {$[7(63)]$} & {$[2.4688]$} & {$[8(72)]$} & {$[2.7969]$} & {$[11(109)]$} & {$[4.2031]$} \\
0.001 & $2(20)$ & 0.9219 & $4(38)$ & 1.5313 & $5(43)$ & 1.6875 \\
& {$[5(46)]$} & {$[1.8438]$} & {$[6(60)]$} & {$[2.4688]$} & {$[10(95)]$} & {$[3.8750]$} \\
0.0001 & $2(18)$ & 0.8594 & $3(27)$ & 1.2500 & $3(28)$ & 1.2656 \\
& {$[4(38)]$} & {$[1.8750]$} & {$[6(56)]$} & {$[2.6875]$} & {$[9(87)]$} & {$[4.1563]$} \\
\hline
\end{tabular}

Table 4: Number and time of iterations of GMRES(10) with preconditioners $\boldsymbol{M}_{t}$ and $\boldsymbol{M}$ for different drop tolerances $\tau, t$ when $h=1 / 32$. Results of preconditioner $\mathcal{M}$ lie inside [ ].

\begin{tabular}{lcccccc}
\hline$\tau$ & $t=-1$ & Time $(-1)$ & $t=1$ & Time $(1)$ & $t=2$ & Time (2) \\
\hline 0.01 & $3(30)$ & 4.0938 & $9(83)$ & 11.4688 & $9(89)$ & 12.1563 \\
& {$[10(93)]$} & {$[12.7344]$} & {$[11(103)]$} & {$[14.1875]$} & {$[13(128)]$} & {$[17.4219]$} \\
0.005 & $3(28)$ & 3.8906 & $7(67)$ & 9.1563 & $8(74)$ & 10.1719 \\
& {$[8(76)]$} & {$[10.4375]$} & {$[9(86)]$} & {$[11.9688]$} & {$[12(118)]$} & {$[16.1719]$} \\
0.001 & $3(22)$ & 3.1719 & $5(44)$ & 6.2813 & $5(45)$ & 6.3438 \\
& {$[6(52)]$} & {$[7.3594]$} & {$[7(65)]$} & {$[9.1719]$} & {$[11(105)]$} & {$[14.7344]$} \\
0.0001 & $2(18)$ & 2.8906 & $3(30)$ & 4.6875 & $4(32)$ & 5.0313 \\
& {$[4(39)]$} & {$[6.0000]$} & {$[6(58)]$} & {$[8.9688]$} & {$[10(94)]$} & {$[14.5000]$} \\
\hline
\end{tabular}

Table 5: Number and time of iterations of GMRES(10) with preconditioners $\widehat{\mathscr{M}}_{t}$ for different drop tolerances $\tau$ and $t$ when $h=1 / 16$.

\begin{tabular}{lcccccccc}
\hline$\tau$ & $t=1 / 2$ & Time $(1 / 2)$ & $t=2$ & Time $(2)$ & $t=4$ & Time $(4)$ & $t=8$ & Time $(8)$ \\
\hline 0.01 & $7(67)$ & 0.4844 & $2(19)$ & 0.1563 & $3(23)$ & 0.2031 & $3(28)$ & 0.2188 \\
0.005 & $6(53)$ & 0.3750 & $2(17)$ & 0.1563 & $2(20)$ & 0.1875 & $3(24)$ & 0.2031 \\
0.001 & $4(38)$ & 0.3125 & $2(14)$ & 0.1406 & $2(16)$ & 0.1719 & $2(17)$ & 0.1563 \\
0.0001 & $4(32)$ & 0.3281 & $2(13)$ & 0.1406 & $2(13)$ & 0.1406 & $2(13)$ & 0.1406 \\
\hline
\end{tabular}

Table 6: Number and time of iterations of GMRES(10) with preconditioners $\widehat{M}_{t}$ for different drop tolerances $\tau$ and $t$ when $h=1 / 24$.

\begin{tabular}{lcccccccc}
\hline$\tau$ & $t=1 / 2$ & Time $(1 / 2)$ & $t=2$ & Time $(2)$ & $t=4$ & Time $(4)$ & $t=8$ & Time $(8)$ \\
\hline 0.01 & $9(87)$ & 3.36 & $3(22)$ & 0.87 & $3(27)$ & 1.03 & $4(34)$ & 1.30 \\
0.005 & $8(79)$ & 3.02 & $2(19)$ & 0.88 & $3(23)$ & 0.90 & $3(28)$ & 1.14 \\
0.001 & $6(52)$ & 2.17 & $2(16)$ & 0.78 & $2(19)$ & 0.78 & $3(22)$ & 1.00 \\
0.0001 & $4(38)$ & 1.77 & $2(14)$ & 0.75 & $2(15)$ & 0.73 & $2(16)$ & 0.83 \\
\hline
\end{tabular}

Table 7: Number and time of iterations of GMRES(10) with preconditioners $\widehat{\mathscr{M}}_{t}$ for different drop tolerances $\tau, t$ when $h=1 / 32$.

\begin{tabular}{lcccccccc}
\hline$\tau$ & $t=1 / 2$ & Time $(1 / 2)$ & $t=2$ & Time $(2)$ & $t=4$ & Time $(4)$ & $t=8$ & Time $(8)$ \\
\hline 0.01 & $11(108)$ & 15.06 & $3(24)$ & 3.39 & $3(28)$ & 3.9 & $4(37)$ & 5.14 \\
0.005 & $9(89)$ & 12.31 & $3(21)$ & 3.02 & $3(24)$ & 3.39 & $3(30)$ & 4.17 \\
0.001 & $6(58)$ & 8.19 & $2(17)$ & 2.47 & $2(20)$ & 2.86 & $3(24)$ & 3.48 \\
0.0001 & $4(39)$ & 6.06 & $2(14)$ & 2.28 & $2(16)$ & 2.55 & $2(17)$ & 2.69 \\
\hline
\end{tabular}




\section{Conclusion}

We have proposed two types of block triangular preconditioners applied to the linear saddle point problems with the singular $(1,1)$ block. The preconditioners have the attractive property of improved eigenvalues clustering with increasing ill-conditioned $(1,1)$ block. The choice of the parameter is involved. Furthermore, according to Corollaries 2.2, 2.4, 2.6, and 2.8, we give the optimal parameter in practice. Numerical experiments are also reported for illustrating the efficiency of the presented preconditioners.

In fact, our methodology can extend the unsymmetrical case; that is, the $(1,2)$ block and the $(2,1)$ block of the saddle point linear system are unsymmetrical.

\section{Acknowledgements}

Warm thanks are due to the anonymous referees and editor Professor Victoria Vampa who made much useful and detailed suggestions that helped the authors to correct some minor errors and improve the quality of the paper. This research was supported by 973 Program (2008CB317110), NSFC $(60973015,10771030)$, the Chinese Universities Specialized Research Fund for the Doctoral Program of Higher Education (20070614001), and the Project of National Defense Key Lab. (9140C6902030906).

\section{References}

[1] A.. Björck, "Numerical stability of methods for solving augmented systems," in Recent Developments in Optimization Theory and Nonlinear Analysis, Y. Censor and S. Reich, Eds., vol. 204 of Contemporary Mathematics, pp. 51-60, American Mathematical Society, Providence, RI, USA, 1997.

[2] S. Wright, "Stability of augmented system factorizations in interior-point methods," SIAM Journal on Matrix Analysis and Applications, vol. 18, no. 1, pp. 191-222, 1997.

[3] H. Elman and D. Silvester, "Fast nonsymmetric iterations and preconditioning for Navier-Stokes equations," SIAM Journal on Scientific Computing, vol. 17, no. 1, pp. 33-46, 1996.

[4] H. C. Elman and G. H. Golub, "Inexact and preconditioned Uzawa algorithms for saddle point problems," SIAM Journal on Numerical Analysis, vol. 31, no. 6, pp. 1645-1661, 1994.

[5] H. C. Elman, D. J. Silvester, and A. J. Wathen, "Iterative methods for problems in computational fluid dynamics," in Iterative Methods in Scientific Computing, R. Chan, T. F. Chan, and G. H. Golub, Eds., pp. 271-327, Springer, Singapore, 1997.

[6] B. Fischer, A. Ramage, D. J. Silvester, and A. J. Wathen, "Minimum residual methods for augmented systems," BIT Numerical Mathematics, vol. 38, no. 3, pp. 527-543, 1998.

[7] C. Li, B. Li, and D. J. Evans, "A generalized successive overrelaxation method for least squares problems," BIT Numerical Mathematics, vol. 38, no. 2, pp. 347-355, 1998.

[8] M. F. Murphy, G. H. Golub, and A. J. Wathen, "A note on preconditioning for indefinite linear systems," Tech. Rep. SCCM-99-03, Stanford University, Stanford, Calif, USA, 1999.

[9] S. G. Nash and A. Sofer, "Preconditioning reduced matrices," SIAM Journal on Matrix Analysis and Applications, vol. 17, no. 1, pp. 47-68, 1996.

[10] B. Fischer, A. Ramage, D. J. Silvester, and A. J. Wathen, "Minimum residual methods for augmented systems," BIT Numerical Mathematics, vol. 38, no. 3, pp. 527-543, 1998.

[11] C. Greif and D. Schötzau, "Preconditioners for the discretized time-harmonic Maxwell equations in mixed form," Numerical Linear Algebra with Applications, vol. 14, no. 4, pp. 281-297, 2007.

[12] T. Rees and C. Greif, "A preconditioner for linear systems arising from interior point optimization methods," SIAM Journal on Scientific Computing, vol. 29, no. 5, pp. 1992-2007, 2007.

[13] J. W. Demmel, Applied Numerical Linear Algebra, SIAM, Philadelphia, Pa, USA, 1997.

[14] Y. Saad, Iterative Methods for Sparse Linear Systems, SIAM, Philadelphia, Pa, USA, 2nd edition, 2003.

[15] C. Greif and D. Schötzau, "Preconditioners for saddle point linear systems with highly singular $(1,1)$ blocks," Electronic Transactions on Numerical Analysis, vol. 22, pp. 114-121, 2006. 
[16] Z.-H. Cao, "Augmentation block preconditioners for saddle point-type matrices with singular $(1,1)$ blocks," Numerical Linear Algebra with Applications, vol. 15, no. 6, pp. 515-533, 2008.

[17] H. C. Elman, "Preconditioning for the steady-state Navier-Stokes equations with low viscosity," SIAM Journal on Scientific Computing, vol. 20, no. 4, pp. 1299-1316, 1999. 


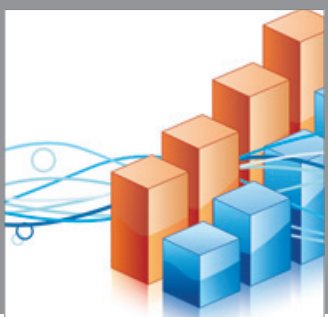

Advances in

Operations Research

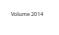

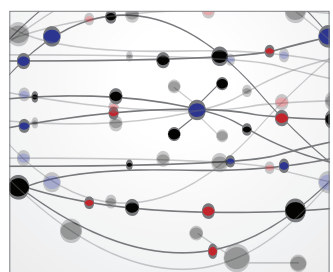

\section{The Scientific} World Journal
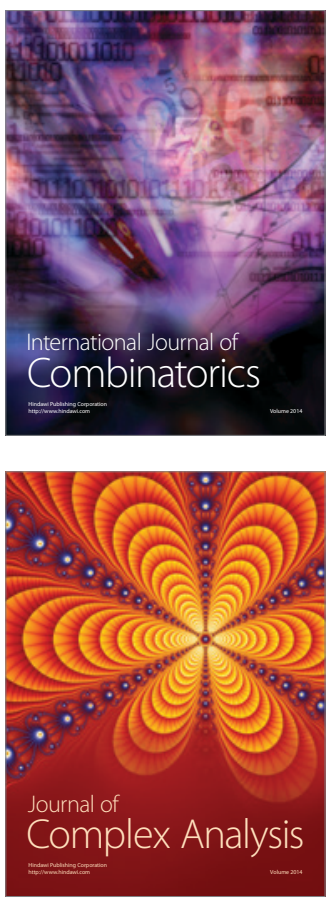

International Journal of

Mathematics and

Mathematical

Sciences
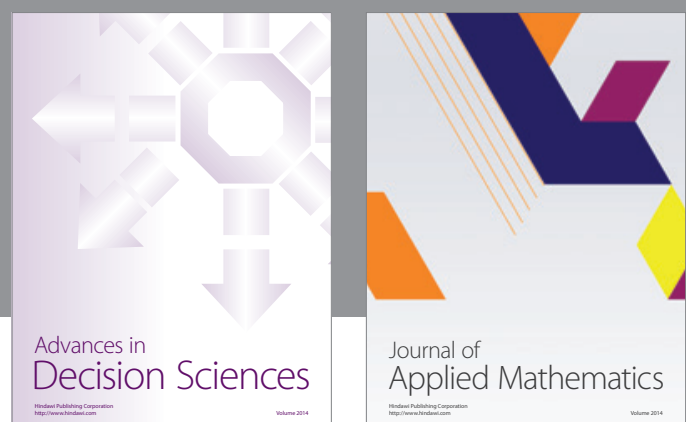

Journal of

Applied Mathematics
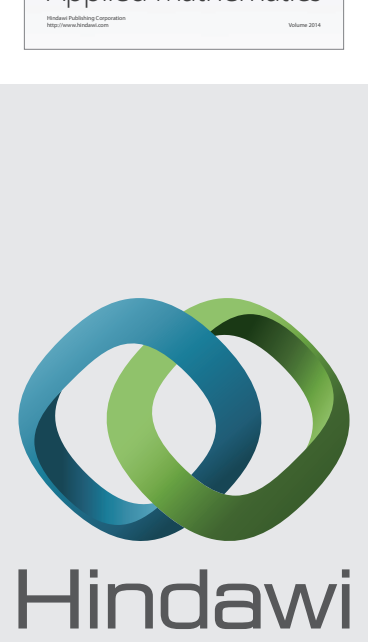

Submit your manuscripts at http://www.hindawi.com
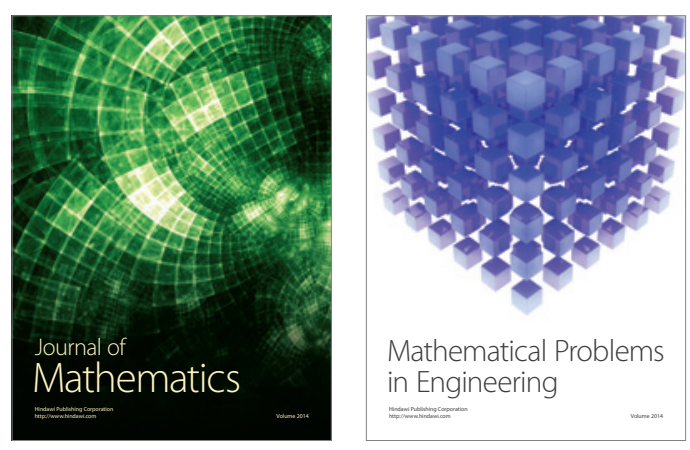

Mathematical Problems in Engineering
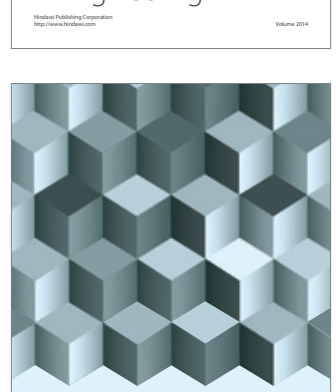

Journal of

Function Spaces
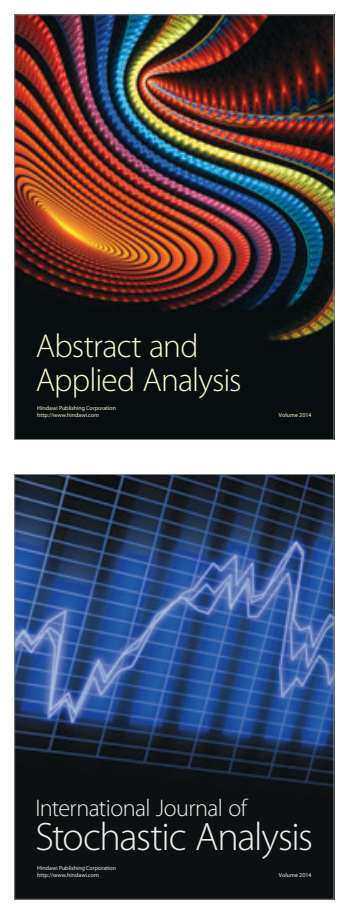

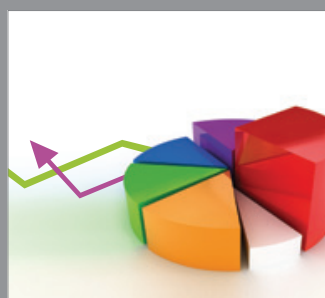

ournal of

Probability and Statistics

Promensencen
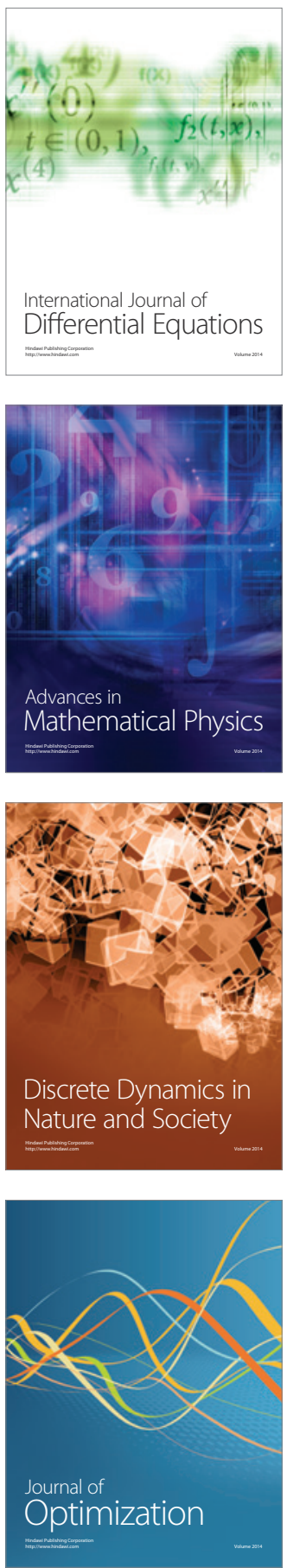